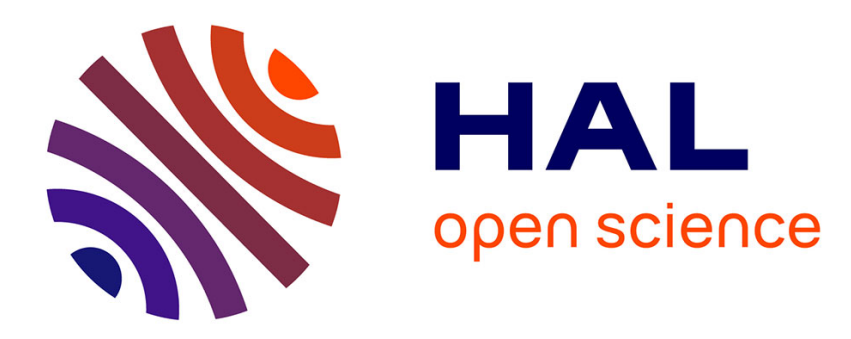

\title{
Teología y política en los villancicos del siglo XVII. El ejemplo de José Pérez de Montoro
}

\author{
Alain Begue
}

\section{To cite this version:}

Alain Begue. Teología y política en los villancicos del siglo XVII. El ejemplo de José Pérez de Montoro. Memoria de la palabra. Actas del VI Congreso de la Asociación Internacional Siglo de Oro, 2004. hal02522964

\section{HAL Id: hal-02522964 \\ https://hal.science/hal-02522964}

Submitted on 14 Apr 2020

HAL is a multi-disciplinary open access archive for the deposit and dissemination of scientific research documents, whether they are published or not. The documents may come from teaching and research institutions in France or abroad, or from public or private research centers.
L'archive ouverte pluridisciplinaire HAL, est destinée au dépôt et à la diffusion de documents scientifiques de niveau recherche, publiés ou non, émanant des établissements d'enseignement et de recherche français ou étrangers, des laboratoires publics ou privés. 


\title{
Teología y política en los villancicos del siglo XVII: el ejemplo de José Pérez de Montoro
}

\author{
Alain Bègue \\ LEMSO, Université de Toulouse-Le Mirail
}

El propósito de la presente ponencia es mostrar cómo el villancico, género poético de función teológico-lúdica, puede convertirse en un recurso propagandístico tanto para la autoridad eclesiástica como para la monarquía. Para ello, nos limitaremos al estudio de los villancicos escritos por el autor setabense José Pérez de Montoro (1627-1694) con motivo de la defensa de la doctrina marial de la Inmaculada Concepción y para la afirmación de la supremacía de la religión católica y de la alabanza de la Casa de Austria, mediante la utilización del contexto histórico de las guerras austro-turcas de los años 1680.

Como bien es sabido, la doctrina de la Inmaculada Concepción goza de una fervorosa devoción popular en España, devoción respaldada por la actuación, desde el siglo XVI, de los monarcas españoles en favor de su dogmatización en Roma. En Andalucía, esta devoción llega incluso a convertirse en defensa encarnizada de la creencia cuando, a principios del siglo XVII, se enciende la ciudad de Sevilla debido a una virulenta disputa entre los dominicos, seguidores de la tesis tomista que excluye a María de todo pecado mortal y venal excepto del original, y las demás órdenes, encabezadas por los franciscanos, máximos defensores de la doctrina ${ }^{1}$. La violencia de los sucesos sevillanos le conducen a Felipe III a que envíe representantes a Roma para que la Santa Sede zanje el problema. Los esfuerzos españoles se ven recompensados con la promulgación, el 8 de diciembre de 1661, de la constitución Sollicitudo omnium Ecclesiarum, que prohíbe cualquier discusión que fuese en contra del sentimiento común y piadoso de la Iglesia ${ }^{2}$. La alegría causada por dicha promulgación en Sevilla queda reflejada en un villancico escrito por Pérez de Montoro ${ }^{3}$.

Junto con Sevilla, Cádiz se destaca por su fuerte implicación en la defensa de la doctrina inmaculista. Esto se explica, según el historiógrafo carmelita Jerónimo de la Concepción, por su primacía universal en el establecimiento y la difusión de la celebración del privilegio mariano. San Basilio, primer obispo de Cádiz y también carmelita, habría sido el instaurador hacia el año 37 de las celebraciones inmaculistas, antes que los demás templos de la Península y mucho antes de que la doctrina fuera adoptada por los demás países ${ }^{4}$. Menos hipotético son el juramento de la defensa de la doctrina marial por los cabildos municipal y catedralicio, en $1618^{5}$, y la petición, el 5 de octubre de 1639, de que cada sermón sea precedido por una alabanza dedicada al «Santísimo Sacramento en nombre de la muy pura concepción de Nuestra Señora concebida sin pecado

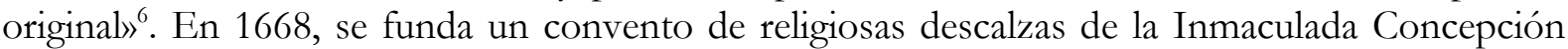
Recoletos ${ }^{7}$ y se publican libros a favor de la doctrina marial ${ }^{8}$. Además, la ciudad portuaria no dejó de hacer suntuosas celebraciones en 1654, después de que Felipe IV hubiera jurado su adhesión a la doctrina, y en 1662, después de la publicación de la bula del papa Alejandro VII ${ }^{9}$.

Así, pues, fue en este contexto cuando Montoro escribió sus treinta y ocho villancicos dedicados a la Inmaculada Concepción, convirtiéndose así en uno de los villanciqueros más fecundos del siglo XVII en el tema. La mayoría de estas obras pertenecen a las siete series elaboradas en Cádiz entre 1688 y 1694, cuando el poeta era el autor villanciqueril oficial de la catedral gaditana. La defensa de la doctrina marial por parte de Montoro se manifiesta

\footnotetext{
${ }^{1}$ Los datos relativos a Sevilla proceden principalmente de Mercedes de los Reyes Peña (2001), pp. 133-143.

${ }^{2}$ Dictionnaire de théologie catholique, tomo 7, primera parte, p. 1175 . En adelante DTC.

${ }^{3} \mathrm{OP}$, tomo 2, pp. 112-113.

${ }^{4}$ Fray Jerónimo de la Concepción, Emporio de el orbe, pp. 206-207.

${ }^{5}$ Archivo Histórico Municipal de Cádiz (AHMC), Actas capitulares, lib. 9, f. 197.

${ }^{6}$ AHMC, Actas capitulares, lib. 20, f. 428.

${ }^{7}$ Arturo Morgado García (1996), pp. 58-59.

${ }^{8}$ Arturo Morgado García (1996), pp. 127-128.

${ }^{9}$ Fray Jerónimo de la Concepción, Emporio de el orbe, Cádiz ilustrada, pp. 465-470.
} 
principalmente en dos planos, el uno semántico-teológico y el otro, estilístico mediante la utilización de un supralenguaje religioso y la inclusión del auditorio en el espacio ficcional de los cantos.

Respecto del primer plano, cabe señalar la insitencia, por parte del poeta, en la imperiosa necesidad de defender el privilegio mariano, mediante su reconocimiento por la Iglesia romana, tal como ocurre en el tercer villancico de la serie de 1693 en el que, aprovechando la estructura dialogada del canto, Montoro crea un debate con fines apologéticos ${ }^{10}$. En el debate, el autor insiste en el hecho de que los feligreses deben seguir la constitución papal que les pide adherir a la doctrina mariana y en la conversión de ésta en dogma católico, al subrayar la diferencia semántica entre los verbos «adorar»y «créen»:

1. De este admirable misterio, principio de nuestro bien, que ardiendo en la caridad, nos ha encendido en la fe, los finos amantes desean saber si es menester, o no es menester, que la ley, que le manda adorar, le manda creer.

2. No es menester.

3. Sí es menester. (OP, II, p. 164, 1693, IC, V3, v. 1-9)

En otras ocasiones, Montoro intenta dar una explicación del privilegio mariano. Así ocurre en el cuarto villancico de la serie de 1688, en el que muestra que dicho privilegio forma parte del programa divino y que María fue preservada del pecado original desde el mismo instante de la falta cometida por Adán y Eva:

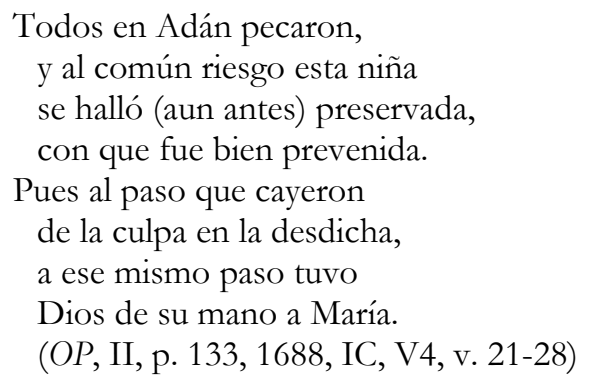

La segunda explicación propugnada por nuestro poeta es la de la pureza de María en el mismo momento de la creación y formación física de su alma, es decir en el primer instante de su concepción tal como lo había defendido Tertuliano al negar, en su obra De carne christi, que la virginidad de María fuera obtenida in partu pero bien ante partum. Se trata de la concepción pasiva, esto es, del momento en que el alma se une con el cuerpo y no de la concepción activa, el acto generador entre san Joaquín y santa Ana ${ }^{11}$.

2. ¿Quién viene allá?

Viene una hermosura recién concebida, que en el mismo instante físico y real de su animación, logró la salud, sin costarla el susto de la enfermedad. (OP, II, p. 125, vv. 8-12)

\footnotetext{
${ }^{10}$ Ya señalamos en otra ocasión que el poeta privilegiaba la estructura de debate en las obras dedicadas a la Inmaculada (Alain Bègue (2001), p. 141).

${ }^{11}$ DTC, tomo 7 , primera parte, p. 846.
} 
La defensa inmaculista pasa asimismo por la utilización de procedimientos estilísticos que consisten principalmente en borrar las fronteras entre el género villanciqueril y otros géneros artísticos, al utilizar el supralenguaje religioso ya existente, y en buscar la participación del auditorio.

El dominio espiritual de la Iglesia hace que se produzca un supralenguaje religioso difundido por los distintos medios artísticos y que refleja una cosmovisión unívoca. En los villancicos de Montoro esto se puede verificar, por ejemplo, en la aproximación del villancico a obras dramáticas religiosas tales como los autos y las loas sacramentales, debida por ejemplo a la introducción de locutores alegóricos. Además, la representación de la Inmaculada en la obra del poeta setabense realza cierta influencia de la iconografía oficial. En efecto, forzoso es constatar que las descripciones de Montoro se conforman — aparte de algunas originalidades al utilizar el hipotexto iconográfico común - a la representación iconográfica de la Inmaculada, que se había fundado desde el siglo XV en los textos bíblicos del Génesis y del Apocalipsis y que se fijan en El arte de la pintura de Francisco Pacheco, publicado en $1649^{12}$. La representación infantil y los atributos de la Inmaculada (túnica blanca, manto azul, corona de doce estrellas, luna y/o una serpiente pisadas por ella) sólo obedecen, en la obra de Montoro, a una finalidad hiperbólica.

Otros recursos estilísticos consisten en hacer partícipe al auditorio, en esfumar la frontera entre el espacio ficcional de las obras y el extraficcional del público. Así, por ejemplo, al esquema estructural fundado en la exhortación inicial, característica del villancico, Montoro le añade en varias ocasiones el empleo de las dos primeras personas del plural. El primer villancico de la serie de 1693 repite a modo de estribillo los versos "Servid y sirvamos, / cantad y cantemos". En este caso, el locutor se dirige al auditorio mediante la utilización de la segunda persona del plural del imperativo para incluirlo después en el espacio textual al emplear la primera persona del plural. Nuestro poeta procede de manera más sutil cuando recurre al presente del indicativo combinado con la primera persona del plural en la modalidad asertiva, como ocurre en el ejemplo siguiente:

1. Con licencia de las luces, hoy, que el ruido de los rayos quiere que el diablo sea sordo, es menester que oiga el diablo: pues si el pobre no supo cómo ni cuándo se encendió la luz bella que hoy adoramos, (OP, II, p. 169, 1693, IC, V5, vv. 1-8)

Montoro supo también poner en práctica estrategias propias de las obras dramáticas, cuando los locutores se convierten en verdaderos personajes que transmiten indirectamente un mensaje al auditorio. El poeta hace así uso del tropo comunicacional que, según lo define Catherine Kerbrat-Orrecchioni, se produce "cada vez que se opera, bajo la presión del contexto una inversión de la jerarquía normal de los destinatarios: es decir cada vez que el destinatario que, en virtud de los indicios de alocución pasa en principio por ser el destinatario directo, no constituye en realidad más que un destinatario segundario, mientras que el verdadero alocutario es en realidad el que tiene en apariencia el estatuto de destinatario indirecto" 13 . En los ejemplos que siguen vemos claramente esta desviación de la trayectoria locutoria hacia el auditorio mediante el uso de frases yusivas implícitas.

4. Sí es menester;

María es merecedora

\footnotetext{
${ }^{12}$ Francisco Pacheco, El arte de la pintura, pp. 575-577.

${ }^{13}$ Catherine Kerbrat-Orecchioni (1998), p. 92.
} 
de lo más, y aunque la den

cuanto obsequioso toca al sacrificio,

siempre es mayor culto el obedecer,

3. Sí es menester,

porque de esta aurora el limpio,

puro, intacto amanecer,

ni duda ya el mundo lo claro que está,

mas con el precepto, sin ojos se ve,

(OP, II, p. 167, 1693, IC, V3, vv. 41-45 y 53-57)

Otro procedimiento radica en la desviación de la función lúdica de las obras conclusivas de las series. Ya señalamos en otro trabajo la gradación interna de las series dedicadas a la Inmaculada Concepción desde una primera composición dedicada a la exhortación y a la majestad del objeto celebrado hasta un último canto político-lúdico en el que se moteja al diablo. Dichas composiciones comparten ciertos rasgos: son jácaras — su tonalidad y música son, pues, ligeras, festivas y cómicas - , se representa burlescamente al diablo —insisten en su derrota y en su aspecto físico infiernal o realzan su estupidez- y su comicidad va acompañada de una violencia que parece tener una función exutoria, catártica para el público. En efecto, a semejanza de lo que ocurre en la conclusión de ciertas obras dramáticas menores, las series villanciqueriles de la Inmaculada concluyen con un canto cuya finalidad es hacer rabiar al personaje "tonto y apaleado". Se multiplican así los verbos y locuciones verbales de acción tales como "sacudir", "raigar el casco", "romper la cabeza", "descalabrar la cholla", "rajar el casquete", "reventar" así como los sustantivos resultativos de violencia ("quebrantahuesos" y "golpe").

Así como utiliza el villancico como medio apologético de la Iglesia católica, al procurar defender la doctrina de la Inmaculada Concepción mediante la explicación del privilegio o recurriendo a procedimientos estilísticos que no tienen como meta sino la reunión del auditorio en torno a la doctrina, Montoro recurre al género villanciqueril como soporte propagandístico al elogiar la Casa de Austria con motivo de las victorias de la coalición cristiana contra los turcos, en los años 1680.

Durante los años 1680, las tropas cristianas reunidas en torno al emperador Leopoldo I de Austria infligen a los otomanos, instalados en Hungría desde su audaz e inaudita invasión de $1529^{14}$, decisivas derrotas y liberan progresivamente el reino de Hungría de su dominio. En 1683, el Gran Visir de Hungría, Kara Mustafá, decide sitiar Viena, la capital imperial, que sólo verá salvación en la intervención de una coalición extranjera encabezada por el duque Carlos $\mathrm{V}$ de Lorena y Juan III Sobieski, rey de Polonia ${ }^{15}$. Tres años después, los principales mandos cristianos concuerdan sitiar Buda, capital de la Hungría turca, que acabará abdicando el 2 de septiembre de 1686 gracias a los esfuerzos conjugados de las tropas del duque de Baviera, Maximiliano Emmanuel, y del ya citado duque de Lorena.

Con este motivo fueron escritos el tercer y el cuarto villancico de la serie de 1683 y las cuatro primeras así como la octava composición de la serie de 1686, interpretadas en el madrileño convento de la Encarnación. Ahora bien, el autor se encuentra con la necesidad de explotar el tema tomando en cuenta el verdadero objeto de la celebración litúrgica: la Natividad. Y esta conciliación se manifiesta a través de la adopción de un carácter narrativo enfatizado fundado en la utilización de géneros y en esquemas estructurales preexistentes.

El primer recurso consiste en la utilización de modos de difusión como las gacetas y relaciones de sucesos, vendidas y narradas por los ciegos. El tercer villancico de la serie de 1683 nos ofrece un buen ejemplo al recrear en la cabeza un diálogo entre dos ciegos, diálogo que debe despertar el interés del auditorio:

\footnotetext{
14 Zoltán Halász (1975), p. 92.

${ }^{15}$ Ungría restavrada..., p. 25.
} 
1. Lleven todos los curiosos

una gaceta nueva

que hoy ha salido.

2. ¿Qué cosa?

3. Prodigios contiene, milagros encierra.

4. Diga de lo que trata.

5. De lo que está pasando en Cielo y tierra.

6. Dénosla luego.

7. Llévenla y crean que es toda un prodigio, por ser verdadera. (OP, II, p. 176, 1683, N, V3, v. 1-13),

Las coplas se abren sucintamente, en un estilo vulgar y con alusiones metafóricas, sobre el nacimiento, la vida de Jesucristo y el significado de su presencia, para relatar a continuación los principales sucesos escogidos por el autor acerca de la batalla de Viena:

1. Lo primero, del Cielo se sabe de aquesta gaceta, que por cierto tropiezo del hombre, Dios ha dado en tierra; y aunque Cordero, un león en Judea dicen le vieron. (OP, II, p. 177, 1683, N, V3, v. 23-29)

Los siete versos de esta estrofa aluden metafóricamente al pecado original (" tropiezo del hombre") que, mediante un concepto fundado en una traslación de los efectos del tropiezo, acarreó la encarnación de Dios ("Dios ha dado en tierra") y la remisión de los pecados por Jesús, hecha posible por el misterio mismo de la Trinidad que permite la traslación de la persona del Padre a la del Hijo, en la base de una doble representación metafórica y antitética de Jesús/«cordero», citado por Juan Bautista y de Jesús/«léon en Judea» del Apocalipsis. La segunda estrofa se apoya en la Pasión de Cristo mediante una alusión eucarística, al recurrir a la socorrida metáfora del grano que simboliza la venida a la tierra del reino de Dios. Montoro resume así a grandes rasgos la historia del Mesías en catorce versos. La estrofa siguiente permite establecer la confluencia de este tema con el de la defensa de Viena. La tradicional asociación de Lucifer con el Islam, fundada en la representación guerrera presentada en el Apocalipsis de una mujer pisando la luna, atributo de la religión musulmana, facilita esta reunión simbólica:

3. Cuenta cómo Luzbel ha caído por esto al infierno,

donde rabia al oír que le llamen

el diablo cojuelo;

Tuvo un enfado, y con él, como en brasas, siempre ha quedado.

4. Cuenta que el Gran Sultán en Turquía, rendido a la Iglesia, ha dispuesto poner los cristianos sobre su cabeza:

Vino por lana, pero le trasquilaron en una plaza.

(OP, II, p. 177, 1683, N, V3, v. 37-50) 
Las diez estrofas que siguen relatan sucesos históricos de la época escogidos por el autor: la invasión de Alemania, la caída de los moros en el Danubio, el abandono de su tienda por el Gran Visir Kara Mustafá en su huída, la persecución de los fugitivos otomanos por los duques de Baviera y Lorena, la coalición de los reyes cristianos, las lágrimas del papa que asiste a la expandión musulmana, y el apoyo del rey polaco Juan III y, por fin, el acta de devoción y de gracias del pueblo español a la Virgen de Atocha.

Además de la elaboración de ciertos villancicos en forma de gaceta, Montoro acude a uno de los personajes tipo que poblaban las obras dramáticas del Siglo de Oro, el morisco. La expulsión definitiva de los moriscos decretada el 4 de abril de 1609 acarreó la menor presencia del personaje en el teatro del siglo XVII pero la coyuntura de los años 1680 favorece su reaparición bien bajo una apariencia noble digna de las novelas bizantinas, bien bajo una apariencia burlesca, como sucede en los villancicos de Montoro. Pero sus personajes no sirven sino para significar su rechazo de la fe mahometana y la superioridad de la religión católica ya que huyen de Buda para refugiarse en Belén. Este rechazo de la religión musulmana los conduce a burlarse de ella y también a justificar la derrota de los moros por el nacimiento de Jesús:

1. Un plaza tan afamada vencer cristianilios pocos.

2. Los turcos, como ser locos, tener cabeza alunada.

1. Un Ninio les dar la entrada, que al moro su madre fía.

2. Por esa Virgen María, e otro Ninio, restaurar. (OP, II, p. 218-219, 1686, N, V3, v. 58-65)

Otro procedimiento consiste en introducir los sucesos húngaros mediante el uso de las representaciones metafóricas tradicionales de la poesía religiosa del siglo XVII desarrolladas en los poemas escritos "en metáfora de", largamente explotados por autores como Alonso de Ledesma o Valdivielso. En el cuarto canto de la serie de 1683 y en el primero de la de 1686, Montoro superpone dos planos, el uno bíblico-alegórico y el otro histórico (la defensa de Viena en el villancico de 1683 y el sitio de Buda en el de 1686). Para ello recurre a la socorrida representación en metáfora de una guerra entre el ejército celestial y las tropas formadas por los ángeles caídos y encabezados por Lucifer.

La ejemplariedad de la composición de 1683 radica en el establecimiento, por parte del autor, de una superposición casi perfecta de ambos planos. Después de la cabeza, en la que unos coros angélicos anuncian a los mortales la nueva victoria celeste y después de haber aludido al nacimiento de Cristo, las coplas relatan las diferentes etapas de la resistencia y luego de la victoria ante el sitio del "enemigo común", esto es, Satanás. El éxito del autor reside en el predominio del plano guerrero aplicado al nacimiento de Cristo y la permanencia de la narración de los sucesos históricos en un plano casi exclusivamente alusivo.

2. Aquel común enemigo, cuya saña vengativa el pueblo de Dios cercado, y en servidumbre tenía:

¡Qué grande dicha

hoy vencidas sus tropas puso en huida!

3. De amotinados rebeldes su ejército componía, a quien seguía en legiones la más abrasada Scitia: 


$$
\text { (OP, II, p. 180, 1683, N, V4, v. 28-38) }
$$

La segunda y la tercera estrofa, que narran el sitio del enemigo, proponen una doble lectura. La alusión al diablo mediante el grupo nominal "enemigo común" era socorrida en la literatura religiosa, pero el contexto histórico permite ver en ella al sultán de Turquía, Mehemet IV, enemigo común de los cristianos que, a través de su representante el Gran Visir, llevó a cabo una lucha encarnizada contra la capital imperial. Asimismo, la alusión metafórica a la esclavitud sufrida por los hombres desde el pecado original resulta ser un tópico literario al que recurre Montoro para simbolizar a la que conocen los habitantes de Viena durante el sitio. En la tercera estrofa, algunos hechos históricos precisos aparecen en filigrana. En 1671 el emperador Leopoldo I intentó imponer un absolutismo centralista al disolver la dieta húngara y al apartar del gobierno a gran parte de la nobleza húngara, provocando así una rebelión encabezada por el joven conde Américo Thököly ${ }^{16}$. Los "amotinados rebeldes" aludidos en el texto son a la vez los ángeles rebelados contra Dios y los bujdosó que se aliaron al Sultán otomano contra el Emperador.

Más adelante, el nacimiento de Cristo es utilizado ingeniosamente por Montoro. La traducción de la encarnación de Dios como abandono del reino celestial por una condición más humilde se convierte, en "metáfora de guerra", en una alusión al abandono de su reino por parte de Juan III de Polonia que se convirtió en soldado liberador de la capital imperial.

6. En este estrecho, un piadoso

Rey por soldado se alista,

y por triunfar de un soberbio,

dejó sus reales insignias:

(OP, II, p. 181, 1683, N, V4, v. 56-59)

Esta superposición se hace explícita en el verso 78 con la creación ingeniosa de un nuevo personaje fundado en la conjunción de los dos planos, bíblico e histórico. La traición de Thököly queda asimilada a la de Judas Iscariote. Sólo el final trágico del apóstol (Mt 27, 5) difiere del refugio y exilio turco del rebelde húngaro ${ }^{17}$.

9. Vendió un traidor a su dueño, que Judas Teklei decían;

pero pagará ahorcado

su infidelidad maligna:

(OP, II, p. 181, 1683, N, V4, v. 77-80)

Con el mismo procedimiento los villancicos de 1686 no dejan de recordar la supremacía de la Casa de Austria. Ya se sabe que gracias a la interpretación de un rezo del profeta Habacuc (Hab 3, 3: "Deus ab austro veniet") y a la aproximación fonética entre el sustantivo latino "austro" y el topónimo "Austria", los Habsburgos se vieron atribuir una función divina. Montoro se apoya tanto en esta asociación como en la comparación de la llegada del viento cálido austral con la venida del Espíritu Santo, hecha a menudo por la Patrística:

2. Alas parece que llevan los invencibles soldados, que al inexpugnable muro valerosos van trepando: ¿Qué mucho, si el César van apellidando, que Sol de las Austrias nos vino del austro?

\footnotetext{
${ }^{16}$ Miguel de Ferdinandi (1967), p. 142-143.

${ }^{17}$ Zoltán Halász (1975), p. 124.
} 
3. ¿Qué feliz!

4. ¿Qué augusto!

5. ¿Qué fuerte!

Preciso es que triunfen,

6. ¡Qué santo!

si en su empeño arduo,

María es la escala, escudo y amparo.

(OP, II, p. 213, 1686 $, \mathrm{N}, \mathrm{V} 1$, v. 67-79)

El emperador se convierte en la encarnación de Dios mediante una metáfora astrológica —el astro solar es uno de los símbolos divinos - y meteorológicos —el viento austral- y, a semejanza de Jesús, beneficia de la protección de María. La incertidumbre del autor en la identificación de los soldados que sitian la ciudad participa también en esta aproximación entre el mundo celeste y el terrestre, aproximación justificada por el autor con la pertenencia del emperador a la Casa de Austria.

La intención panegírica del autor aparece también en la descripción, en el cuarto villancico de la serie de 1686, del acto de gracias hecho por Carlos II a la Virgen de Atocha en celebración de la conquista de Buda. La narración ofrece a nuestro autor la posibilidad de representar a la familia real a través del prisma metafórico religioso. El enfermizo monarca se ve atribuida una imagen crística al convertirse en medio de sus cortesanos en el Rey divino, imagen avalada por la función divina medieval atribuida a los reyes.

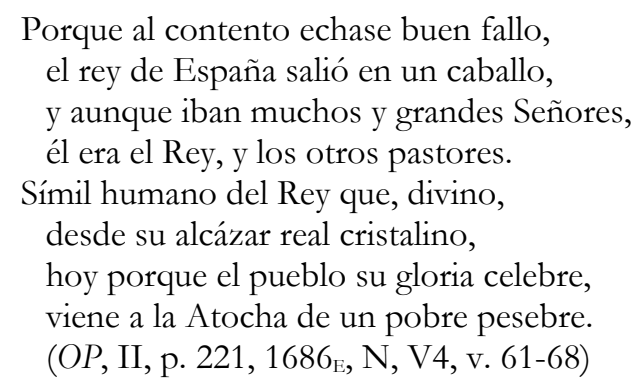

Esta representación se funda en la comparación directa establecida en el sintagma binominal "símil (...) del Rey" que viene a reforzar el adjetivo calificativo "humano" y dos metáforas: el reino divino se convierte, mediante una metáfora in absentia, en el alcázar del soberano español mientras que el monasterio de Atocha queda asimilado, gracias a una metáfora fundada en un sustantivo complemento de nombre, en el pesebre del Niño Jesús. Para acabar, y de manera análoga, veamos que el sintagma lexicalizado que se refiere a la madre del rey ("reina madre") sirve de punto de partida para situar a Mariana de Austria y a la Virgen María en un mismo plano. Al conferirle, además de su función maternal, una función matrimonial, el autor crea una relación metafórica que se desprenderá nítidamente en la aplicación de motivos solares generalmente asociados a la Inmaculada Concepción ("alba", "aurora ” y "sol ”).

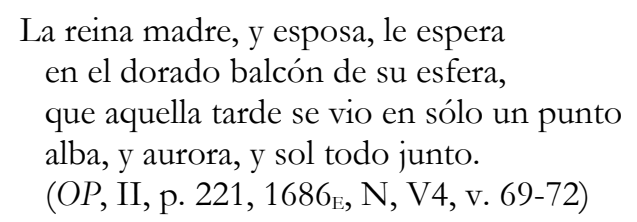

Hemos intentado destacar en el presente estudio algunos de los procedimientos empleados por Pérez de Montoro con fines doctrinales y políticos, en la medida que se trataba de apoyar desde el género villanciqueril la política religiosa llevada a cabo tanto por la Iglesia como por la monarquía española a través de los ejemplos concretos de la defensa de la doctrina de la 
Inmaculada Concepción y de la celebración de dos victorias cristianas en las guerras austro-turcas de los años 1680, a saber la defensa de la ciudad de Viena en 1683 y la toma de la capital de la Hungría turca, Buda, en 1686. Dichos procedimientos no hacen sino subrayar una vez más el carácter camaleónico y polimórfico de este género complejo, completo y ejemplar que es el villancico barroco.

\section{Bibliografía}

Bègue, Alain, «La primera réplica en los villancicos polifónicos de José Pérez de Montoro», Criticón, 83, 2001, pp. 133-146.

Dictionnaire de théologie catholique contenant l'exposé des doctrines de la théologie catholique, leurs preuves et leur bistoire, Paris, Librairie Letouzey et Ané, 1922.

Ferdinandi, Miguel de, Historia de Hungría, Madrid, Alianza, 1967 (Libro de bolsillo, 46).

Halász, Zoltán, Historia de Hungría, Editorial Corvina, 1975.

Jerónimo de la Concepción, Fray, Emporio de el orbe, Cadiz ilustrada, investigacion de sus antiguas Grandezas, discurridas en concurso de el general imperio de España, Amsterdam, Juan Bus, 1690.

Kerbrat-Orecchioni, Catherine, Les interactions verbales. 1/ Approche interactionnelle et structure des conversations, Paris, Armand Colin, 1998 (U "Linguistique").

Morgado García, Arturo, El estamento eclesiástico y la vida espiritual en la diócesis de Cádiz en el siglo XVII, Cádiz, Servicio de Publicaciones de la Universidad de Cádiz, 1996.

Pacheco, Francisco, El arte de la pintura, ed., intr. y notas de Bonaventura Bassegodas i Hugas, Madrid, Cátedra, 1990 (Arte. Grandes Temas).

Pérez de Montoro, José, Obras posthumas lyricas, Madrid, Antonio Marín, 1736. 2 tomos.

Reyes Peña, Mercedes de los, "Un pasquín anti-inmaculista en la Sevilla del primer tercio del siglo XVII", en Rogelio Reyes Cano, Mercedes de los Reyes Peña y Klaus Wagner (eds.), Sevilla y la literatura. Homenaje al profesor Francisco López. Estrada en su 80 cumpleaños, Sevilla, Secretariado de Publicaciones de la Universidad de Sevilla, 2001 (Literatura, 46), pp. 133-160. 